\title{
A Clinical Study of the Evaluation and Assessment of the Etiology and Patterns of Ocular Injuries in Midfacial Trauma in a Tertiary Care Hospital
}

\author{
Shrikar Umarane ${ }^{1}$, Tejraj Kale ${ }^{1}$, Arvind Tenagi ${ }^{2}$, Yash Manavadaria ${ }^{1}$, Abhishek S. Motimath Sr. ${ }^{1}$ \\ 1. Oral and Maxillofacial Surgery, KLE Vishwanath Katti Institute of Dental Sciences, KLE Academy of Higher \\ Education and Research, Belagavi, IND 2. Ophthalmology, Jawaharlal Nehru Medical College, KLE Academy of Higher \\ Education and Research, Belagavi, IND
}

Corresponding author: Yash Manavadaria, dryashmanavadaria@gmail.com

\section{Abstract}

\section{Aims}

The aim is to study the pattern of ocular injuries in midfacial trauma and to evaluate the overall incidence of ophthalmic injury of any severity following maxillofacial trauma.

\section{Methods and Materials}

The maxillofacial surgeon conducted routine facial examination of patients with midfacial fractures, which also included a detailed ophthalmologic examination of patients, at the time of initial presentation. These patients were then further evaluated by an ophthalmologist for thorough examination of the eye.

\section{Results}

The total number of recorded midface maxillofacial trauma cases was 181 . Out of 181 patients, 161 had ocular injuries. Among 181 cases, 161 (88.95\%) cases were due to road traffic accidents, which was the prime etiologic factor. Out of total 181 patients, 172 (95.03\%) were males and 9 (4.97\%) were females. The maximum number of cases were of zygomaticomaxillary complex fractures (44.75\%) followed by nasal bone fractures (21.5\%). Periorbital ecchymosis accounted for the maximum number of cases, amounting to $61.88 \%$. Loss of vision or blindness was seen in eight $(4.42 \%)$ patients.

\section{Conclusions}

The study stresses further on the importance of long-term and continuous data collection and record management of trauma patients, which may help health care providers with necessary information to develop treatment protocols and device measures for the prevention of complications.

Received 08/19/2020

Review began 08/24/2020

Review ended 08/27/2020

Published 09/02/2020

(c) Copyright 2020

Umarane et al. This is an open access article distributed under the terms of the Creative Commons Attribution License CC-BY 4.0., which permits unrestricted use, distribution, and reproduction in any medium, provided the original author and source are credited.
Categories: Ophthalmology, Trauma

Keywords: midfacial fractures, ocular injuries, maxillofacial injuries, eye signs

\section{Introduction}

Maxillofacial fractures account for a substantial proportion in traumatology [1]. The severity of injuries ranges from a simple abrasion on the skin to tissue loss and complex facial fractures leading to cosmetic and functional discrepancy. Therefore, maxillofacial injuries may have a great amount of emotional, psychological, and financial impact on the patient because of the eventual disability and disfigurement.

The etiology of the trauma differs considerably according to the geographic location, culture, and socioeconomic status of the population. Road traffic accidents (RTAs) continue to be the leading cause, with assault coming close second. Recent studies indicated toward RTAs being the most frequent etiology in developing countries, whereas assault was the main cause in developed nations. Fall happens to be the most frequent cause of maxillofacial injuries in children and elderly individuals [1,2].

Several authors note that the most common site of maxillofacial fractures is the midfacial bones [3]. Maxillofacial trauma is often associated with serious concomitant injuries. Apart from the grave and lifethreatening associated injuries, injury to the vital structures that the maxillofacial skeleton houses may lead to severe disability.

"Vision", an important prime sense relies on the eye, is an organ occupying only $0.3 \%$ of total body surface [3]. In spite of the built-in protection (anatomy), ocular injuries causing significant functional and 
aesthetic defects are associated with 6-94\% of maxillofacial injuries. Ocular injuries often accompany midface injuries. All facial trauma injuries, particularly above the level of the mouth, require a careful ophthalmic examination [4].

The degree of severity of ocular injuries to the eye and its adjacent structures varies a lot. The injuries range from contused lacerated wounds of the eyelids and corneal abrasions to wounds or rupture of the sclera, dislocation of the lens, intraorbital hemorrhages, and detachment of the retina. Globe rupture, optic nerve damage, derangement of the visual pathway as a result of retrobulbar hemorrhage, and perineural edema causing nerve compression subsequently leading to ischemic optic neuropathy remain to be the most commonly occurring ocular injuries. Hence, facial injuries particularly those affecting the midface warrant a careful examination of the eye by a specialist trauma team and eye surgeons.

The time of initial examination may be the only time when injuries to the vital structures, such as the retina and optic nerve, may be evident because later during the course of time, these signs become inconspicuous due to continuous hemorrhage. Some ocular injuries require immediate simultaneous surgical intervention of a maxillofacial surgeon and an ophthalmologist, whereas others may just render the immediate maxillofacial surgery contraindicated $[4,5]$.

Last but not the least, recognition of an ocular injury before operation is important from a medico-legal point of view, as it ensures that the repair is not later attributed to be the cause of any permanent visual disturbance [4].

Few studies show a strong correlation between zygomaticomaxillary complex (ZMC) fractures and ocular injuries, whereas others point toward higher a incidence of ocular injuries with Le Fort III fractures. The prevalence of ocular injuries associated with facial fractures has been widely reported to be $2.7 \%$ to $90.6 \%$. Overall, $95 \%$ of severe ocular injuries are associated with fractures of the facial middle third $[6,7]$.

This descriptive study was designed to give a general idea of orbital fractures and ocular injuries associated with maxillofacial trauma. This will be a valuable aid in an early diagnosis and management of ocular injuries in midfacial trauma, which is important for the prevention of ocular dysfunction.

\section{Materials And Methods \\ Study population}

The study population consisted of 181 patients who reported to KLES Dr. Prabhakar Kore Hospital and Research Centre, Belagavi, Karnataka, India. The hospital is one of the tertiary referral centers for Belagavi district apart from other private and government institutes with a population of around 4.8 million according to the census of 2011.

\section{Inclusion criteria}

All patients with midfacial trauma, with or without other facial bone fractures who reported to KLES Dr. Prabhakar Kore Hospital and Research Centre in the period of September 2016 to May 2018 were included in the study after getting approval from the Ethical Committee of our institute.

\section{Exclusion criteria}

Patients reporting with pre-existing congenital or acquired ophthalmic disease or infections, ocular disorders such as cataract, glaucoma, and retinal disorders, and age-related macular degeneration (ARMD) were not a part of the study. This study did not include patients who received treatment in other health care facility apart from primary repair. Patients who were brought dead to the hospital or died during the course of treatment and those who were not willing for treatment and wanted to leave against medical advice were also excluded from the study.

\section{Data collection}

Simple random sampling technique was used for data collection. After a written informed consent from the patient was taken, maintaining the confidentiality, a pre-structured and pre-tested questionnaire was used to gather information followed by a thorough ocular examination. In cases where the condition of the victims did not warrant the interview, the relatives or attendants were interviewed. Medical records and case sheets were referred whenever necessary to collect additional information.

\section{Statistical analysis}

The data collected were analyzed using chi-square test and stratified according to age, sex, etiology, fracture type, and pattern of ocular injury.

\section{Procedure}




\section{Cureus}

The maxillofacial surgeon conducted routine maxillofacial and oral examination of patients with clinically and radiographically proven midfacial fractures, which also included a detailed ophthalmologic examination of patients at the time of initial presentation. These patients were then further evaluated by an ophthalmologist for thorough examination of the eye.

\section{Results}

The maxillofacial trauma was divided into ZMC fractures, Le Fort I, II, and III fractures, nasoorbitoethmoidal (NOE) fractures, orbital fractures, nasal fractures, zygomatic arch fractures, frontal bone fractures, and palatal fractures.

\section{Etiology}

Among 181 patients, RTAs were the primary cause of midfacial fractures (88.95\%) (Table 1).

\begin{tabular}{|c|c|c|}
\hline Injuries & Number & Percentage \\
\hline RTA & 161 & 88.95 \\
\hline Assault & 12 & 6.63 \\
\hline Fall & 5 & 2.76 \\
\hline Others & 3 & 1.66 \\
\hline Total & 181 & 100.00 \\
\hline
\end{tabular}

TABLE 1: Distribution of patients according to etiology of the midfacial fractures.

RTA, road traffic accidents

Within the category of RTAs, motorcycle accidents accounted for 115 (71.43\%) cases followed by collisions with light and heavy vehicles that included 30 (18.63\%) cases, and bicycle/bullock cart/pedestrian accidents that accounted for 16 (9.94\%) cases (Table 2).

\begin{tabular}{|l|l|}
\hline Type of RTA & Number \\
\hline Motorcycle accident & $115(161)$ \\
\hline Four-wheeler/bus/heavy vehicle & $30(161)$ \\
\hline Bicycle/bullock cart/pedestrian hit & $16(161)$ \\
\hline
\end{tabular}

TABLE 2: Type of the RTA in patients with midface fracture.

RTA, road traffic accidents

Upto $28.73 \%$ of patients were under the influence of alcohol at the time of injury, of which 52 were males.

\section{Age distribution}

Out of 181 patients, 66 (36.46\%) were in the third decade of life and accounted for the maximum percentage of maxillofacial injuries followed by fourth (36 patients [19.89\%]) and fifth decade (35 patients [19.34\%]) of life. Patients below 20 years of age were less commonly affected, accounting only for $10.50 \%$ of the total. Only $4.42 \%$ of patients were from the age group of above 60 years (Table 3). 


\section{Cureus}

\begin{tabular}{|l|l|l|}
\hline Age groups & Number & Percentage \\
\hline$\leq 20$ years & 19 & 10.50 \\
\hline $21-30$ years & 66 & 36.46 \\
\hline $31-40$ years & 36 & 19.89 \\
\hline $41-50$ years & 35 & 19.34 \\
\hline $51-60$ years & 17 & 9.39 \\
\hline$\geq 61$ years & 8 & 4.42 \\
\hline Total & 181 & 100.00 \\
\hline Mean age & 35.15 & \\
\hline SD age & 13.61 & \\
\hline
\end{tabular}

TABLE 3: Age-wise distribution of fractures.

The right side was frequently affected, and ZMC fracture was the most commonly found fracture (48 patients [26.52\%]) (Table 4). 


\section{Cureus}

\begin{tabular}{|c|c|c|}
\hline Fractures & Number & Percentage \\
\hline R nasal & 12 & 6.63 \\
\hline L nasal & 6 & 3.31 \\
\hline B/L nasal & 21 & 11.60 \\
\hline R ZMC & 48 & 26.52 \\
\hline L ZMMC & 29 & 16.02 \\
\hline LFI & 6 & 3.31 \\
\hline RLFI & 0 & 0.00 \\
\hline LLF 1 & 1 & 0.55 \\
\hline LFII & 10 & 5.52 \\
\hline RLF\| & 4 & 2.21 \\
\hline L LF II & 3 & 1.66 \\
\hline LF III & 7 & 3.87 \\
\hline R LF III & 2 & 1.10 \\
\hline L LF III & 0 & 0.00 \\
\hline $\mathrm{R}$ roof of the orbit & 7 & 3.87 \\
\hline$L$ roof of the orbit & 11 & 6.08 \\
\hline R floor of the orbit & 9 & 4.97 \\
\hline$L$ floor of the orbit & 3 & 1.66 \\
\hline R lateral wall of the orbit & 6 & 3.31 \\
\hline L lateral wall of the orbit & 5 & 2.76 \\
\hline $\mathrm{R}$ medial wall of the orbit & 3 & 1.66 \\
\hline$L$ medial wall of the orbit & 1 & 0.55 \\
\hline R zygomatic arch & 21 & 11.60 \\
\hline L zygomatic arch & 15 & 8.29 \\
\hline Frontal bone & 29 & 16.02 \\
\hline Palate & 9 & 4.97 \\
\hline NOE & 4 & 2.21 \\
\hline
\end{tabular}

\section{TABLE 4: Fracture-wise distribution.}

R, right; L, left; B/L, bilateral; ZMC, zygomaticomaxillary complex; LF, Le Fort; NOE, nasoorbitoethmoidal

ZMC fractures were commonly seen in the third decade followed by the fourth and fifth decades. Most of the frontal bone fractures were also seen in the third decade of life (Table 5). 


\begin{tabular}{|c|c|c|c|c|c|c|c|c|c|c|}
\hline Fractures & $\begin{array}{l}\leq 20 \\
\text { years }\end{array}$ & $\begin{array}{l}21-30 \\
\text { years }\end{array}$ & $\begin{array}{l}31-40 \\
\text { years }\end{array}$ & $\begin{array}{l}41-50 \\
\text { years }\end{array}$ & $\begin{array}{l}51-60 \\
\text { years }\end{array}$ & $\begin{array}{l}\geq 61 \\
\text { years }\end{array}$ & Total & $\%$ & $\begin{array}{l}\text { Chi- } \\
\text { square }\end{array}$ & $\begin{array}{l}\text { p- } \\
\text { Value }\end{array}$ \\
\hline R nasal & 4 & 2 & 3 & 1 & 2 & 0 & 12 & 6.63 & 10.0320 & 0.0740 \\
\hline L nasal & 1 & 3 & 0 & 1 & 0 & 1 & 6 & 3.31 & 4.4830 & 0.4820 \\
\hline B/L nasal & 3 & 9 & 3 & 3 & 1 & 2 & 21 & 11.60 & 3.2220 & 0.6660 \\
\hline R ZMC & 3 & 17 & 12 & 8 & 6 & 2 & 48 & 26.52 & 2.9220 & 0.7120 \\
\hline L ZMC & 4 & 13 & 3 & 7 & 2 & 0 & 29 & 16.02 & 4.7680 & 0.4450 \\
\hline LFI & 0 & 2 & 0 & 3 & 0 & 1 & 6 & 3.31 & 7.6080 & 0.1790 \\
\hline R LFI & 0 & 0 & 0 & 0 & 0 & 0 & 0 & 0.00 & -- & -- \\
\hline L LF 1 & 0 & 0 & 1 & 0 & 0 & 0 & 1 & 0.55 & 4.0500 & 0.5420 \\
\hline LF II & 1 & 6 & 2 & 0 & 1 & 0 & 10 & 5.52 & 4.1290 & 0.5310 \\
\hline R LF II & 0 & 2 & 1 & 1 & 0 & 0 & 4 & 2.21 & 1.3210 & 0.9330 \\
\hline LLF\| & 0 & 2 & 1 & 0 & 0 & 0 & 3 & 1.66 & 2.3720 & 0.7960 \\
\hline LFF III & 1 & 2 & 1 & 1 & 2 & 0 & $r$ & 3.87 & 3.6090 & $0.60 / 0$ \\
\hline R LF III & 1 & 0 & 1 & 0 & 0 & 0 & 2 & 1.10 & 5.3360 & 0.3760 \\
\hline L LF III & 0 & 0 & 0 & 0 & 0 & 0 & 0 & 0.00 & -- & -- \\
\hline $\mathrm{R}$ roof of the orbit & 0 & 2 & 1 & 2 & 1 & 1 & 7 & 3.87 & 3.1140 & 0.6820 \\
\hline$L$ roof of the orbit & 0 & 3 & 4 & 3 & 1 & 0 & 11 & 6.08 & 3.9990 & 0.5500 \\
\hline$R$ floor of the orbit & 0 & 4 & 1 & 2 & 0 & 2 & 9 & 4.97 & 9.2480 & 0.1000 \\
\hline$L$ floor of the orbit & 0 & 0 & 0 & 2 & 1 & 0 & 3 & 1.66 & 7.5700 & 0.1820 \\
\hline $\begin{array}{l}\mathrm{R} \text { lateral wall of the } \\
\text { orbit }\end{array}$ & 0 & 0 & 2 & 2 & 2 & 0 & 6 & 3.31 & 8.1680 & 0.1470 \\
\hline $\begin{array}{l}\text { L lateral wall of the } \\
\text { orbit }\end{array}$ & 1 & 0 & 2 & 1 & 0 & 1 & 5 & 2.76 & 6.6710 & 0.2460 \\
\hline $\begin{array}{l}\mathrm{R} \text { medial wall of the } \\
\text { orbit }\end{array}$ & 1 & 0 & 0 & 1 & 1 & 0 & 3 & 1.66 & 5.5400 & 0.3540 \\
\hline $\begin{array}{l}\text { medial wall of the } \\
\text { orbit }\end{array}$ & 0 & 0 & 0 & 0 & 1 & 0 & 1 & 0.55 & 9.7010 & 0.0840 \\
\hline R zygomatic arch & 2 & 4 & 7 & 3 & 5 & 0 & 21 & 11.60 & 10.7770 & 0.0560 \\
\hline L zygomatic arch & 1 & 5 & 2 & 4 & 1 & 2 & 15 & 8.29 & 4.1500 & 0.5280 \\
\hline Frontal bone & 2 & 16 & 3 & 3 & 4 & 1 & 29 & 16.02 & 7.5530 & 0.1830 \\
\hline Palate & 2 & 4 & 1 & 1 & 1 & 0 & 9 & 4.97 & 2.5530 & 0.7690 \\
\hline NOE & 0 & 3 & 0 & 0 & 1 & 0 & 4 & 2.21 & 4.9410 & 0.4230 \\
\hline
\end{tabular}

TABLE 5: Age range distribution of subjects as per the type of midfacial fracture.

R, right; L, left; B/L, bilateral, ZMC, zygomaticomaxillary complex; LF, Le Fort; NOE, nasoorbitoethmoidal

\section{Sex distribution}

Out of total 181 patients, 172 (95.03\%) were found to be males and 9 (4.97\%) females. Most of the maxillofacial trauma cases were seen among males (Table 6). 


\section{Cureus}

\begin{tabular}{|c|c|c|}
\hline Gender & Number & Percentage \\
\hline Male & 172 & 95.03 \\
\hline Female & 9 & 4.97 \\
\hline Total & 181 & 100.00 \\
\hline
\end{tabular}

TABLE 6: Gender-wise distribution of midfacial fractures.

Maximum number of males sustained fractures in the third decade of life (64 patients), whereas females (3 patients) were more in the fifth decade of life (Table 7).

\begin{tabular}{|c|c|c|c|c|c|}
\hline Age groups & Male & $\%$ & Female & $\%$ & Number \\
\hline$\leq 20$ years & 17 & 89.47 & 2 & 10.53 & 19 \\
\hline $21-30$ years & 64 & 96.97 & 2 & 3.03 & 66 \\
\hline $31-40$ years & 35 & 97.22 & 1 & 2.78 & 36 \\
\hline $41-50$ years & 32 & 91.43 & 3 & 8.57 & 35 \\
\hline $51-60$ years & 16 & 94.12 & 1 & 5.88 & 17 \\
\hline$\geq 61$ years & 8 & 100.00 & 0 & 0.00 & 8 \\
\hline Total & 172 & 95.03 & 9 & 4.97 & 181 \\
\hline
\end{tabular}

TABLE 7: Age-wise distribution of males and females who sustained midfacial fractures.

Table 8 shows the distribution of males and females in different types of fractures. Maximum number of cases were of ZMC fractures (44.75\%), accounting for a total of 73 cases in males and 4 cases in females. In the midface, nasal bone fracture cases were second highest in number, accounting for 39 patients. 


\begin{tabular}{|c|c|c|c|c|c|c|}
\hline Fractures & Male & Female & Total & $\%$ & Chi-square & p-Value \\
\hline R nasal & 12 & 0 & 12 & 6.63 & 0.6720 & 0.4120 \\
\hline L nasal & 6 & 0 & 6 & 3.31 & 0.3250 & 0.5690 \\
\hline B/L nasal & 21 & 0 & 21 & 11.60 & 1.2430 & 0.2650 \\
\hline R ZMC & 47 & 1 & 48 & 26.52 & 1.1540 & 0.2830 \\
\hline L ZMC & 26 & 3 & 29 & 16.02 & 2.1090 & 0.1460 \\
\hline LF I & 6 & 0 & 6 & 3.31 & 0.3250 & 0.5690 \\
\hline R LF I & 0 & 0 & 0 & 0.00 & -- & -- \\
\hline L LF 1 & 1 & 0 & 1 & 0.55 & 0.0530 & 0.8190 \\
\hline LF II & 10 & 0 & 10 & 5.52 & 0.5540 & 0.4570 \\
\hline R LF II & 3 & 1 & 4 & 2.21 & 3.4720 & 0.0620 \\
\hline LLF II & 3 & 0 & 3 & 1.66 & 0.1600 & 0.6900 \\
\hline LF III & 7 & 0 & 7 & 3.87 & 0.3810 & 0.5370 \\
\hline R LF III & 2 & 0 & 2 & 1.10 & 0.1060 & 0.7450 \\
\hline L LF III & 0 & 0 & 0 & 0.00 & -- & -- \\
\hline $\mathrm{R}$ roof of the orbit & 7 & 0 & 7 & 3.87 & 0.3810 & 0.5370 \\
\hline $\mathrm{L}$ roof of the orbit & 10 & 1 & 11 & 6.08 & 0.4200 & 0.5170 \\
\hline $\mathrm{R}$ floor of the orbit & 9 & 0 & 9 & 4.97 & 0.4960 & 0.4810 \\
\hline$L$ floor of the orbit & 3 & 0 & 3 & 1.66 & 0.1600 & 0.6900 \\
\hline $\mathrm{R}$ lateral wall of the orbit & 6 & 0 & 6 & 3.31 & 0.3250 & 0.5690 \\
\hline L lateral wall of the orbit & 4 & 1 & 5 & 2.76 & 2.4580 & 0.1170 \\
\hline $\mathrm{R}$ medial wall of the orbit & 3 & 0 & 3 & 1.66 & 0.1600 & 0.6900 \\
\hline L medial wall of the orbit & 1 & 0 & 1 & 0.55 & 0.0530 & 0.8190 \\
\hline R zygomatic arch & 20 & 1 & 21 & 11.60 & 0.0020 & 0.9620 \\
\hline L zygomatic arch & 14 & 1 & 15 & 8.29 & 0.0990 & 0.7530 \\
\hline Frontal bone & 29 & 0 & 29 & 16.02 & 1.8070 & 0.1790 \\
\hline Palate & 9 & 0 & 9 & 4.97 & 0.4960 & 0.4810 \\
\hline NOE & 4 & 0 & 4 & 2.21 & 0.2140 & 0.6440 \\
\hline
\end{tabular}

TABLE 8: Association between genders with midfacial fractures.

R, right; L, left; B/L, bilateral; ZMC, zygomaticomaxillary complex; LF, Le Fort; NOE, nasoorbitoethmoidal

\section{Pattern of ocular injuries}

Figure 1 shows the number of patients associated with different types of ocular injuries. Periorbital ecchymosis accounted for the maximum number of cases, adding up to $61.88 \%$, followed by periorbital edema and subconjunctival hemorrhage. Chemosis was seen in $25.41 \%$, vitreous hemorrhage in $0.55 \%$, traumatic optic neuropathy in $11.05 \%$, corneal injury in $1.66 \%$, reduced acuity in $5.52 \%$, traumatic telecanthus in $1.66 \%$, retrobulbar hemorrhage in $0.55 \%$, diplopia in $1.66 \%$, enophthalmos in $1.66 \%$ and hyphema in $0.55 \%$. Globe rupture was seen in $1.66 \%$ and intraorbital hemorrhage in $1.10 \%$. Retinal detachment was seen in $0.55 \%$, lens subluxation in $0.55 \%$, Berlin's edema in $0.55 \%$, and uveal, lens, and vitreous incarceration was seen in two (1.10\%) cases. Proptosis and traumatic mydriasis occurred only in two (1.10\%) patients and 1 (0.55\%) patient, respectively. Traumatic III nerve palsy was seen in one patient and canalicular injuries were seen in three (1.66\%) patients. Eyelid lacerations were seen in 25 (13.81\%) cases. 


\section{Cureus}

Loss of vision or blindness was seen in eight (4.42\%) patients and was associated with different fracture patterns.

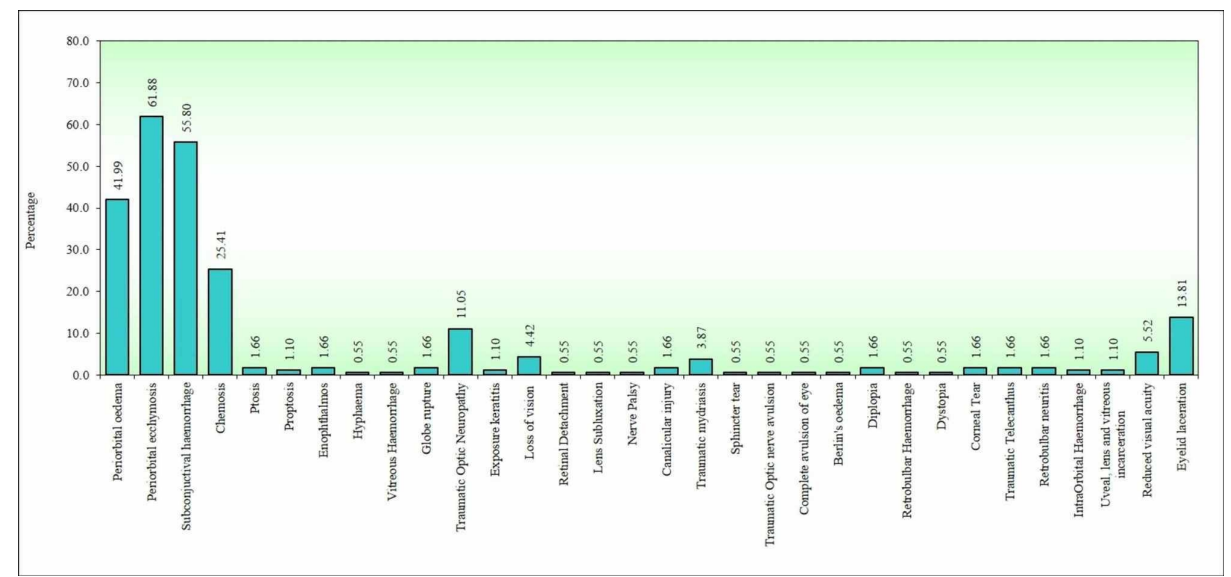

FIGURE 1: Ocular Injuries-wise distribution.

${ }^{*} \mathrm{p}<0.05$

Most of the ocular injuries were seen in males (Table 9). 


\section{Cureus}

\begin{tabular}{|c|c|c|c|c|}
\hline Injuries & Male & Female & Chi-square & $\mathrm{p}$-Value \\
\hline Periorbital edema & 70 & 6 & 2.3680 & 0.1240 \\
\hline Periorbital ecchymosis & 106 & 6 & 0.0920 & 0.7620 \\
\hline Subconjunctival hemorrhage & 97 & 4 & 0.4950 & 0.4820 \\
\hline Chemosis & 45 & 1 & 1.0220 & 0.3120 \\
\hline Ptosis & 3 & 0 & 0.1600 & 0.6900 \\
\hline Proptosis & 2 & 0 & 0.1060 & 0.7450 \\
\hline Enophthalmos & 3 & 0 & 0.1600 & 0.6900 \\
\hline Hyphema & 1 & 0 & 0.0530 & 0.8190 \\
\hline Vitreous hemorrhage & 1 & 0 & 0.0530 & 0.8190 \\
\hline Globe rupture & 3 & 0 & 0.1600 & 0.6900 \\
\hline Traumatic optic neuropathy & 20 & 0 & 1.1770 & 0.2780 \\
\hline Exposure keratitis & 2 & 0 & 0.1060 & 0.7450 \\
\hline Loss of vision & 7 & 1 & 1.0040 & 0.3160 \\
\hline Retinal detachment & 1 & 0 & 0.0530 & 0.8190 \\
\hline Lens subluxation & 1 & 0 & 0.0530 & 0.8190 \\
\hline Nerve palsy & 1 & 0 & 0.0530 & 0.8190 \\
\hline Canalicular injury & 3 & 0 & 0.1600 & 0.6900 \\
\hline Traumatic mydriasis & 7 & 0 & 0.3810 & 0.5370 \\
\hline Sphincter tear & 1 & 0 & 0.0530 & 0.8190 \\
\hline Traumatic optic nerve avulsion & 1 & 0 & 0.0530 & 0.8190 \\
\hline Complete avulsion of eye & 1 & 0 & 0.0530 & 0.8190 \\
\hline Berlin's edema & 1 & 0 & 0.0530 & 0.8190 \\
\hline Diplopia & 3 & 0 & 0.1600 & 0.6900 \\
\hline Retrobulbar hemorrhage & 1 & 0 & 0.0530 & 0.8190 \\
\hline Dystopia & 1 & 0 & 0.0530 & 0.8190 \\
\hline Corneal tear & 3 & 0 & 0.1600 & 0.6900 \\
\hline Traumatic telecanthus & 3 & 0 & 0.1600 & 0.6900 \\
\hline Retrobulbar neuritis & 3 & 0 & 0.1600 & 0.6900 \\
\hline Intraorbital hemorrhage & 2 & 0 & 0.1060 & 0.7450 \\
\hline Uveal, lens, and vitreous incarceration & 2 & 0 & 0.1060 & 0.7450 \\
\hline Reduced visual acuity & 10 & 0 & 0.5540 & 0.4570 \\
\hline Eyelid laceration & 25 & 0 & 1.5180 & 0.2180 \\
\hline
\end{tabular}

TABLE 9: Ocular injury and gender distribution. 


\begin{tabular}{|c|c|c|c|c|c|c|c|c|}
\hline Injuries & $\begin{array}{l}\leq 20 \\
\text { years }\end{array}$ & $\begin{array}{l}21-30 \\
\text { years }\end{array}$ & $\begin{array}{l}31-40 \\
\text { years }\end{array}$ & $\begin{array}{l}41-60 \\
\text { years }\end{array}$ & $\begin{array}{l}51-60 \\
\text { years }\end{array}$ & $\begin{array}{l}\geq 61 \\
\text { years }\end{array}$ & $\begin{array}{l}\text { Chi- } \\
\text { square }\end{array}$ & $\begin{array}{l}\mathrm{p}- \\
\text { Value }\end{array}$ \\
\hline Periorbital edema & 6 & 28 & 15 & 16 & 9 & 2 & 2.8360 & 0.7250 \\
\hline Periorbital ecchymosis & 13 & 44 & 19 & 21 & 12 & 3 & 4.8650 & 0.4330 \\
\hline Subconjunctival hemorrhage & 12 & 37 & 18 & 21 & 10 & 3 & 2.3100 & 0.8050 \\
\hline Chemosis & 5 & 18 & 4 & 10 & 5 & 4 & 6.8920 & 0.2290 \\
\hline Ptosis & 0 & 3 & 0 & 0 & 0 & 0 & 5.3150 & 0.3790 \\
\hline Proptosis & 0 & 0 & 0 & 0 & 0 & 2 & 43.7330 & $0.0001^{*}$ \\
\hline Enophthalmos & 1 & 2 & 0 & 0 & 0 & 0 & 3.8970 & 0.5640 \\
\hline Hyphema & 0 & 1 & 0 & 0 & 0 & 0 & 1.7520 & 0.8820 \\
\hline Vitreous hemorrhage & 0 & 0 & 0 & 0 & 0 & 0 & - & - \\
\hline Globe rupture & 0 & 0 & 3 & 0 & 0 & 0 & 12.2870 & $0.0310^{*}$ \\
\hline Traumatic optic neuropathy & 2 & 9 & 2 & 2 & 1 & 4 & 15.3840 & $0.0090^{*}$ \\
\hline Exposure keratitis & 0 & 2 & 0 & 0 & 0 & 0 & 3.5240 & 0.6200 \\
\hline Loss of vision & 0 & 4 & 1 & 1 & 1 & 1 & 3.0540 & 0.6920 \\
\hline Retinal detachment & 0 & 0 & 0 & 0 & 0 & 0 & - & - \\
\hline Lens subluxation & 0 & 0 & 0 & 0 & 0 & 0 & - & - \\
\hline Nerve palsy & 0 & 1 & 0 & 0 & 0 & 0 & 1.7520 & 0.8820 \\
\hline Canalicular injury & 2 & 0 & 0 & 0 & 0 & 1 & 17.5340 & $0.0040^{*}$ \\
\hline Traumatic mydriasis & 0 & 2 & 1 & 1 & 1 & 2 & 10.8950 & 0.0540 \\
\hline Sphincter tear & 0 & 0 & 0 & 0 & 0 & 0 & - & - \\
\hline Traumatic optic nerve avulsion & 0 & 0 & 1 & 0 & 0 & 0 & 4.0500 & 0.5420 \\
\hline Complete avulsion of eye & 0 & 0 & 1 & 0 & 0 & 0 & 4.0500 & 0.5420 \\
\hline Berlin's edema & 0 & 0 & 0 & 0 & 1 & 0 & 9.7010 & 0.0840 \\
\hline Diplopia & 0 & 2 & 0 & 0 & 0 & 1 & 8.3360 & 0.1390 \\
\hline Retrobulbar hemorrhage & 0 & 0 & 0 & 0 & 0 & & - & - \\
\hline Dystopia & 0 & 0 & 0 & 1 & 0 & 0 & 4.1950 & 0.5220 \\
\hline Corneal tear & 0 & 1 & 0 & 1 & 1 & 0 & 3.2410 & 0.6630 \\
\hline Traumatic telecanthus & 0 & 3 & 0 & 0 & 0 & 0 & 5.3150 & 0.3790 \\
\hline Retrobulbar neuritis & 1 & 0 & 2 & 0 & 0 & 0 & 6.9950 & 0.2210 \\
\hline Intraorbital hemorrhage & 0 & 1 & 0 & 0 & 0 & 1 & 10.8030 & 0.0550 \\
\hline $\begin{array}{l}\text { Uveal, lens, and vitreous } \\
\text { incarceration }\end{array}$ & 0 & 0 & 2 & 0 & 0 & 0 & 8.1460 & 0.1480 \\
\hline Reduced visual acuity & 1 & 5 & 0 & 1 & 0 & 3 & 19.7810 & $0.0010^{*}$ \\
\hline Eyelid laceration & 2 & 9 & 6 & 4 & 4 & 0 & 3.2180 & 0.6660 \\
\hline
\end{tabular}

TABLE 10: Association between age groups and ocular injuries.

${ }^{*} p<0.05$.

Tables 11-13 show the distribution of ocular injuries in different types of fractures. 


\begin{tabular}{|c|c|c|c|c|c|c|c|c|c|c|c|c|c|c|}
\hline Fractures & $\begin{array}{l}R \\
\text { nasal }\end{array}$ & nasal & $\begin{array}{l}\mathrm{B} / \mathrm{L} \\
\text { nasal }\end{array}$ & $\begin{array}{l}\text { R } \\
\text { ZMC }\end{array}$ & ZMC & $\begin{array}{l}\text { LF } \\
\text { I }\end{array}$ & $\begin{array}{l}\text { R } \\
\text { LF I }\end{array}$ & $\begin{array}{l}\mathrm{L} \\
\mathrm{LF} I\end{array}$ & $\begin{array}{l}\text { LF } \\
\text { II }\end{array}$ & $\begin{array}{l}\text { R LF } \\
\text { II }\end{array}$ & $\begin{array}{l}\text { L LF } \\
\text { II }\end{array}$ & $\begin{array}{l}\text { LF } \\
\text { III }\end{array}$ & $\begin{array}{l}\text { R LF } \\
\text { III }\end{array}$ & $\begin{array}{l}\text { L LF } \\
\text { III }\end{array}$ \\
\hline Periorbital edema & 5 & 2 & 5 & 23 & 14 & 2 & 0 & 1 & 8 & 3 & 3 & 2 & 1 & 0 \\
\hline Periorbital ecchymosis & 7 & 3 & 11 & 31 & 25 & 2 & 0 & 1 & 8 & 3 & 3 & 6 & 2 & 0 \\
\hline Subconjunctival hemorrhage & 4 & 3 & 5 & 31 & 22 & 3 & 0 & 1 & 8 & 3 & 2 & 6 & 2 & 0 \\
\hline Chemosis & 5 & 2 & 3 & 12 & 7 & 3 & 0 & 0 & 2 & 1 & 1 & 2 & 0 & 0 \\
\hline Ptosis & 0 & 0 & 0 & 0 & 0 & 0 & 0 & 0 & 0 & 0 & 0 & 0 & 0 & 0 \\
\hline Proptosis & 0 & 0 & 0 & 0 & 0 & 0 & 0 & 0 & 0 & 0 & 0 & 0 & 0 & 0 \\
\hline Enophthalmos & 0 & 0 & 0 & 0 & 1 & 0 & 0 & 0 & 0 & 0 & 0 & 1 & 0 & 0 \\
\hline Hyphema & 0 & 0 & 0 & 0 & 0 & 0 & 0 & 0 & 0 & 0 & 0 & 1 & 0 & 0 \\
\hline Vitreous hemorrhage & 0 & 0 & 0 & 0 & 0 & 0 & 0 & 0 & 0 & 0 & 0 & 1 & 0 & 0 \\
\hline Globe rupture & 2 & 0 & 0 & 1 & 0 & 0 & 0 & 0 & 0 & 0 & 0 & 0 & 0 & 0 \\
\hline Traumatic optic neuropathy & 1 & 2 & 3 & 5 & 2 & 0 & 0 & 0 & 1 & 1 & 1 & 3 & 0 & 0 \\
\hline Exposure keratitis & 0 & 0 & 0 & 1 & 0 & 0 & 0 & 0 & 0 & 0 & 0 & 1 & 0 & 0 \\
\hline Loss of vision & 1 & 0 & 1 & 3 & 0 & 0 & 0 & 1 & 0 & 0 & 0 & 0 & 1 & 0 \\
\hline Retinal detachment & 0 & 0 & 0 & 0 & 0 & 0 & 0 & 0 & 0 & 0 & 0 & 0 & 1 & 0 \\
\hline Lens subluxation & 0 & 0 & 0 & 0 & 0 & 0 & 0 & 0 & 0 & 0 & 0 & 0 & 1 & 0 \\
\hline Nerve palsy & 0 & 0 & 0 & 1 & 0 & 0 & 0 & 0 & 0 & 0 & 0 & 0 & 0 & 0 \\
\hline Canalicular injury & 1 & 0 & 1 & 0 & 0 & 0 & 0 & 0 & 0 & 0 & 0 & 0 & 0 & 0 \\
\hline Traumatic mydriasis & 1 & 1 & 0 & 3 & 1 & 0 & 0 & 0 & 0 & 0 & 0 & 1 & 0 & 0 \\
\hline Sphincter tear & 0 & 0 & 0 & 0 & 0 & 0 & 0 & 0 & 0 & 0 & 0 & 0 & 1 & 0 \\
\hline Traumatic optic nerve avulsion & 0 & 0 & 0 & 1 & 0 & 0 & 0 & 0 & 0 & 0 & 0 & 0 & 0 & 0 \\
\hline Complete avulsion of eye & 0 & 0 & 0 & 1 & 0 & 0 & 0 & 0 & 0 & 0 & 0 & 0 & 0 & 0 \\
\hline Berlin's edema & 0 & 0 & 0 & 0 & 0 & 0 & 0 & 0 & 1 & 0 & 0 & 0 & 0 & 0 \\
\hline Diplopia & 0 & 0 & 0 & 1 & 0 & 0 & 0 & 0 & 0 & 0 & 0 & 0 & 0 & 0 \\
\hline Retrobulbar hemorrhage & 0 & 0 & 0 & 0 & 0 & 0 & 0 & 0 & 0 & 0 & 0 & 0 & 0 & 0 \\
\hline Dystopia & 0 & 0 & 0 & 0 & 0 & 0 & 0 & 0 & 0 & 0 & 0 & 0 & 0 & 0 \\
\hline Corneal tear & 0 & 0 & 0 & 0 & 0 & 0 & 0 & 0 & 1 & 0 & 0 & 0 & 0 & 0 \\
\hline Traumatic telecanthus & 0 & 1 & 0 & 1 & 0 & 0 & 0 & 0 & 0 & 0 & 0 & 0 & 0 & 0 \\
\hline Retrobulbar neuritis & 2 & 0 & 0 & 1 & 0 & 0 & 0 & 0 & 0 & 0 & 0 & 0 & 0 & 0 \\
\hline Intraorbital hemorrhage & 0 & 0 & 0 & 0 & 0 & 0 & 0 & 0 & 0 & 0 & 0 & 1 & 0 & 0 \\
\hline $\begin{array}{l}\text { Uveal, lens, and vitreous } \\
\text { incarceration }\end{array}$ & 2 & 0 & 0 & 0 & 0 & 0 & 0 & 0 & 0 & 0 & 0 & 0 & 0 & 0 \\
\hline Reduced visual acuity & 1 & 1 & 1 & 0 & 1 & 0 & 0 & 0 & 0 & 0 & 0 & 1 & 0 & 0 \\
\hline Eyelid laceration & 5 & 1 & 4 & 4 & 0 & 0 & 0 & 0 & 1 & 0 & 0 & 2 & 0 & 0 \\
\hline
\end{tabular}

TABLE 11: Association between fractures and ocular injuries.

R, right; L, left; B/L, bilateral; ZMC, zygomaticomaxillary complex; LF, Le Fort 


\section{Cureus}

\begin{tabular}{|c|c|c|c|c|c|c|c|c|}
\hline Fractures & $\begin{array}{l}R \text { roof of } \\
\text { the orbit }\end{array}$ & $\begin{array}{l}L \text { roof of } \\
\text { the orbit }\end{array}$ & $\begin{array}{l}\text { R floor of } \\
\text { the orbit }\end{array}$ & $\begin{array}{l}L \text { floor of } \\
\text { the orbit }\end{array}$ & $\begin{array}{l}\mathrm{R} \text { lateral wall } \\
\text { of the orbit }\end{array}$ & $\begin{array}{l}\text { L lateral wall } \\
\text { of the orbit }\end{array}$ & $\begin{array}{l}\text { R medial wall } \\
\text { of the orbit }\end{array}$ & $\begin{array}{l}\text { L medial wall } \\
\text { of the orbit }\end{array}$ \\
\hline Periorbital edema & 3 & 4 & 2 & 1 & 3 & 1 & 1 & 0 \\
\hline $\begin{array}{l}\text { Periorbital } \\
\text { ecchymosis }\end{array}$ & 4 & 6 & 3 & 1 & 2 & 3 & 0 & 0 \\
\hline $\begin{array}{l}\text { Subconjunctival } \\
\text { hemorrhage }\end{array}$ & 2 & 7 & 4 & 0 & 5 & 3 & 2 & 0 \\
\hline Chemosis & 3 & 4 & 3 & 1 & 3 & 2 & 1 & 0 \\
\hline Ptosis & 1 & 0 & 1 & 0 & 0 & 0 & 0 & 0 \\
\hline Proptosis & 0 & 0 & 2 & 0 & 0 & 0 & 0 & 0 \\
\hline Enophthalmos & 1 & 0 & 1 & 0 & 0 & 0 & 0 & 0 \\
\hline Hyphema & 0 & 0 & 0 & 0 & 0 & 0 & 0 & 0 \\
\hline Vitreous hemorrhage & 0 & 0 & 0 & 0 & 0 & 0 & 0 & 0 \\
\hline Globe rupture & 0 & 0 & 0 & 0 & 0 & 0 & 0 & 0 \\
\hline $\begin{array}{l}\text { Traumatic optic } \\
\text { neuropathy }\end{array}$ & 0 & 2 & 2 & 1 & 0 & 1 & 0 & 0 \\
\hline Exposure keratitis & 0 & 0 & 0 & 0 & 0 & 0 & 0 & 0 \\
\hline Loss of vision & 0 & 1 & 1 & 0 & 0 & 0 & 0 & 0 \\
\hline Retinal detachment & 0 & 0 & 0 & 0 & 0 & 0 & 0 & 0 \\
\hline Lens subluxation & 0 & 0 & 0 & 0 & 0 & 0 & 0 & 0 \\
\hline Nerve palsy & 0 & 0 & 0 & 0 & 0 & 0 & 0 & 0 \\
\hline Canalicular injury & 0 & 0 & 1 & 0 & 0 & 0 & 0 & 0 \\
\hline Traumatic mydriasis & 2 & 0 & 1 & 1 & 1 & 0 & 0 & 0 \\
\hline Sphincter tear & 0 & 0 & 0 & 0 & 0 & 0 & 0 & 0 \\
\hline $\begin{array}{l}\text { Traumatic optic } \\
\text { nerve avulsion }\end{array}$ & 0 & 0 & 0 & 0 & 0 & 0 & 0 & 0 \\
\hline $\begin{array}{l}\text { Complete avulsion of } \\
\text { eye }\end{array}$ & 0 & 0 & 0 & 0 & 0 & 0 & 0 & 0 \\
\hline Berlin's edema & 0 & 0 & 0 & 0 & 0 & 0 & 0 & 0 \\
\hline Diplopia & 1 & 0 & 1 & 0 & 0 & 0 & 0 & 0 \\
\hline $\begin{array}{l}\text { Retrobulbar } \\
\text { hemorrhage }\end{array}$ & 0 & 0 & 0 & 0 & 0 & 0 & 0 & 0 \\
\hline Dystopia & 0 & 0 & 1 & 0 & 0 & 0 & 0 & 0 \\
\hline Corneal tear & 0 & 0 & 1 & 0 & 0 & 0 & 0 & 0 \\
\hline $\begin{array}{l}\text { Iraumatic } \\
\text { telecanthus }\end{array}$ & 0 & 0 & 0 & 0 & 0 & 0 & 0 & 0 \\
\hline Retrobulbar neuritis & 0 & 0 & 0 & 0 & 0 & 0 & 0 & 0 \\
\hline $\begin{array}{l}\text { Intraorbital } \\
\text { hemorrhage }\end{array}$ & 0 & 0 & 1 & 0 & 0 & 0 & 0 & 0 \\
\hline $\begin{array}{l}\text { Uveal, lens, and } \\
\text { vitreous } \\
\text { incarceration }\end{array}$ & 0 & 0 & 0 & 0 & 0 & 0 & 0 & 0 \\
\hline $\begin{array}{l}\text { Reduced visual } \\
\text { acuity }\end{array}$ & 1 & 0 & 2 & 1 & 0 & 0 & 0 & 0 \\
\hline Eyelid laceration & 1 & 2 & 1 & 1 & 1 & 1 & 0 & 0 \\
\hline
\end{tabular}




\section{Cureus}

TABLE 12: Association between fractures and ocular injuries.

$R$, right; L, left 


\section{Cureus}

\begin{tabular}{|c|c|c|c|c|c|}
\hline Fractures & R zygomatic arch & L zygomatic arch & Frontal bone & Palate & NOE \\
\hline Periorbital edema & 8 & 4 & 10 & 4 & 1 \\
\hline Periorbital ecchymosis & 9 & 8 & 19 & 6 & 2 \\
\hline Subconjunctival hemorrhage & 4 & 5 & 16 & 6 & 1 \\
\hline Chemosis & 3 & 4 & 7 & 3 & 1 \\
\hline Ptosis & 1 & 0 & 1 & 0 & 1 \\
\hline Proptosis & 0 & 0 & 0 & 0 & 0 \\
\hline Enophthalmos & 0 & 0 & 1 & 0 & 0 \\
\hline Hyphema & 0 & 0 & 0 & 0 & 0 \\
\hline Vitreous hemorrhage & 0 & 0 & 0 & 0 & 0 \\
\hline Globe rupture & 0 & 0 & 2 & 0 & 0 \\
\hline Traumatic optic neuropathy & 0 & 4 & 4 & 1 & 1 \\
\hline Exposure keratitis & 0 & 0 & 0 & 0 & 0 \\
\hline Loss of vision & 0 & 2 & 1 & 0 & 0 \\
\hline Retinal detachment & 0 & 0 & 0 & 0 & 0 \\
\hline Lens subluxation & 0 & 0 & 0 & 0 & 0 \\
\hline Nerve palsy & 0 & 0 & 0 & 0 & 0 \\
\hline Canalicular injury & 0 & 0 & 0 & 0 & 0 \\
\hline Traumatic mydriasis & 2 & 1 & 0 & 1 & 0 \\
\hline Sphincter tear & 0 & 0 & 0 & 0 & 0 \\
\hline Traumatic optic nerve avulsion & 0 & 0 & 0 & 0 & 0 \\
\hline Complete avulsion of eye & 0 & 0 & 0 & 0 & 0 \\
\hline Berlin's edema & 0 & 0 & 0 & 0 & 0 \\
\hline Diplopia & 0 & 0 & 1 & 0 & 0 \\
\hline Retrobulbar hemorrhage & 0 & 0 & 0 & 0 & 0 \\
\hline Dystopia & 0 & 0 & 0 & 0 & 0 \\
\hline Corneal tear & 0 & 0 & 1 & 0 & 1 \\
\hline Traumatic telecanthus & 0 & 0 & 2 & 0 & 2 \\
\hline Retrobulbar neuritis & 0 & 0 & 2 & 0 & 0 \\
\hline Intraorbital hemorrhage & 0 & 0 & 1 & 0 & 0 \\
\hline Uveal, lens, and vitreous incarceration & 0 & 0 & 2 & 0 & 0 \\
\hline Reduced visual acuity & 0 & 1 & 3 & 0 & 1 \\
\hline Eyelid laceration & 2 & 2 & 11 & 2 & 3 \\
\hline
\end{tabular}

TABLE 13: Association between fractures and ocular injuries.

R, right; L, left; NOE, nasoorbitoethmoidal

Maximum number of ocular injuries were seen in right ZMC fractures followed by left ZMC fractures and frontal bone fractures (Tables 11-13). 
In the ZMC fracture cases treated for open reduction and internal fixation, there was no statistically significant difference between the heart rate pre-operatively and intra-operatively (Table 14).

\begin{tabular}{|c|c|c|c|c|c|c|}
\hline Time points & Mean & SD & Mean difference & SD difference & Paired t-test & p-Value \\
\hline Pre-operative & 71.80 & 3.57 & & & & \\
\hline At the time of reduction of fracture & 72.16 & 3.20 & -0.36 & 3.98 & -0.7777 & 0.4392 \\
\hline Pre-operative & 71.80 & 3.57 & & & & \\
\hline Post-operative & 72.26 & 3.19 & -0.46 & 3.94 & -1.0178 & 0.3120 \\
\hline At the time of reduction of fracture & 72.16 & 3.20 & & & & \\
\hline Post-operative & 72.26 & 3.19 & -0.11 & 4.67 & -0.1964 & 0.8448 \\
\hline
\end{tabular}

TABLE 14: Heart rate pre-operative, intra-operative, and post-operative of ZMC fractures.

ZMC, zygomaticomaxillary complex

\section{Discussion}

Orbital injury may lead to few of the most important complications following a maxillofacial trauma as it increases the risk of ocular trauma and optic nerve injury. The utmost importance of early diagnosis and intervention has been greatly highlighted by several studies particularly when there are definitive signs and symptoms of retrobulbar hemorrhage and increased intraocular tension. Even though the eye is fairly well protected against trauma by several factors such as eyelids, orbital rim, reflex actions such as blinking and hand over eye or head rotation away from the impact, facial fractures especially orbital fractures may still subject the patient at a risk of ocular injury $[5,6]$.

The midfacial skeleton comprises the maxilla, frontal bone, zygoma, zygomatic arch, palatine bone, vomer, and nasal bones. Sphenoid, frontal, and ethmoid bones may not be part of facial structures anatomically but are frequently involved in midfacial fractures. Midfacial fractures are commonly classified as Le Fort I, II, and III fractures, ZMC, zygomatic arch fractures, NOE fractures, nasal bone fractures, and fractures of the palate and frontal bone. These may occur in isolation or together $[1,6]$.

Ocular injuries in association with facial trauma can be attributed to variable etiology. These can be due to sports, industrial hazards, or assault, and can also be self-inflicted. In all high-speed RTAs, the injuries usually are grave and lead to orbital fractures and eventually injury to the eyeball and associated structures. Having a sound knowledge of association of ocular injuries and its types and frequency plays a pivotal role in giving a wholesome treatment to the patient. If the diagnosis is missed, it will influence the overall treatment and ultimate prognosis of the patient $[5,6,8]$.

The demographic data and pattern of maxillofacial fractures vary according to various socioeconomic and geographic factors $[2,8]$. When it comes to etiology, RTAs remain to be the leading cause of maxillofacial trauma $[9,10]$. Studies conducted in developed nations have suggested a changing trend in the etiology of maxillofacial trauma from RTA to assault, with fall being the most common etiology $[9,10]$. Septa et al. reported the incidence or maxillofacial trauma due to RTA to be 64\%. In RTA, two-wheelers appear to be the major cause of accident, with accidents due to motorcycles accounting for 55.4\% [8]. A total of 181 patients with midfacial fractures were included in our study. In our study, 115 cases (71.43\%) of RTA due to twowheelers were seen followed by four-wheelers and other heavy vehicles (18.63\%) and 16 cases wherein the victim was a pedestrian or in a bullock cart (16 patients [9.94\%]). Septa et al. reported that $33.5 \%$ of patients were under alcohol influence, with males accounting for 99\% [8]. In our study, 28.73\% were under the influence of alcohol and all of them were males ( 52 patients). Thus, there should be strong pressure by governments through advertisement and television to outlaw drunk drivers and enforce regulation.

Majority of the maxillofacial trauma cases were seen in patients in the third decade of life followed by fourth and fifth decades. First and seventh decades of life accounted for least number of cases. These findings are supported by various studies in literature suggesting maxillofacial injuries to be more common in the second and third decades of life [11,12]. Low incidence of maxillofacial injuries have also been reported by some authors in age groups below 10 years and above 60 years $[13,14]$.

Of the total patients recorded, $95.03 \%$ were male and $4.97 \%$ were females. The ratio of males to females was 19.11:1. These values in other studies show that the ratio ranged from 2.19:1 to as high as 11.8:1. Males are shown to be more susceptible to maxillofacial trauma in almost all the previous studies $[15,16]$. 
Most common midfacial fracture recorded in our study was of ZMC (44.75\%) followed by nasal fractures (21.5\%). Le I, II, and III fractures accounted for 3.86\%, 9.39\%, and 3.86\%, respectively. Frontal bone fractures accounted for $16.02 \%$. NOE fractures accounted for $2.21 \%$. ZMC fractures comprising the maximum number of cases is a finding that is concurrent with other studies $[1,16,17]$. The incidence of Le Fort fractures reported by us was quite low compared to studies conducted by other authors [18-20].

Occurrence of concomitant ocular injuries with maxillofacial fractures may range from as low as $2.7 \%$ to as high as $90 \%$ [21-24]. In our study, out of the total 181 patients, 161 (88.9\%) patients had some type of ocular injuries. It falls within the range documented by other authors as mentioned above. Such a wide range of reported incidence of concomitant ocular injuries maybe due to difference in types of ocular injuries included in the study and difference in expertise of the examiner and specificity of the examination performed.

Periorbital ecchymosis (61.88\%) accounted for the maximum number of total ocular injuries followed by periorbital edema (41.99\%) and subconjunctival hemorrhage (55.80\%). Chemosis was seen in $25.41 \%$, vitreous hemorrhage in $0.55 \%$, traumatic optic neuropathy in $11.05 \%$, corneal injury in $1.66 \%$, reduced acuity in $5.52 \%$, traumatic telecanthus in $1.66 \%$, retrobulbar hemorrhage in $0.55 \%$, diplopia in $1.66 \%$, enophthalmos in $1.66 \%$ and hyphema in $0.55 \%$. Globe rupture was seen in $1.66 \%$ and intraorbital hemorrhage in $1.10 \%$. Retinal detachment was seen in $0.55 \%$, lens subluxation in $0.55 \%$, Berlin's edema in $0.55 \%$, and uveal, lens, and vitreous incarceration in $1.10 \%$. Proptosis and traumatic mydriasis occurred only in two $(1.10 \%)$ patients and one $(0.55 \%)$ patient, respectively. Traumatic III nerve palsy was seen in one patient, canalicular injuries in three (1.66\%) patients, and eyelid lacerations in 25 (13.81\%) patients. Loss of vision or blindness was seen in eight (4.42\%) patients.

Subconjunctival hemorrhage being one of the most common ocular injuries in our study compares favorably with other studies, where it is found to be between 60 and $74 \%[25,26]$. Of the total 181 cases of subconjunctival hemorrhage, 101 (55.80\%) were found in association with ZMC fractures, Le Fort fractures, frontal bone fractures, nasal bone fractures, and orbital fractures.

Diplopia is one of the common findings in patients of maxillofacial fractures. The incidence of diplopia was found to be $1.66 \%$ in our study, which is significantly less than the incidence reported by other studies, which was around $19 \%$ [25,27,28]. Septa et al. reported $11.5 \%$ patients with diplopia [8]. Mittal et al. reported a $60 \%$ incidence of diplopia in orbital fractures, and rest $40 \%$ were seen in ZMC fractures [5]. In our study, ZMC fractures, orbital floor fractures, and orbital roof fractures were associated with diplopia cases.

Enophthalmos following a maxillofacial trauma is a definitive indication of radiological imaging to confirm fractures of orbital wall. CT scan being more specific is advised over plain X-ray. In our study, a total of $1.66 \%$ patients presented with enophthalmos, which is on the lower side when compared to figures (8\%) reported by Al-Qurainy et al. [28]. Septa et al. reported an incidence of $8.5 \%$ for enophthalmos [8]. Amrith et al. recorded a $6 \%$ incidence of enophthalmos, with $81 \%$ of it occurring in orbital fracture patients [22]. In our study, out of three cases of enophthalmos, one was associated with orbital floor and roof fractures, one was associated with ZMC fractures, and one was associated with Le Fort III fractures.

All patients with maxillofacial injuries should mandatorily be checked for pupillary reflex and visual acuity. These are often called as "vital signs" of the eye. We found reduced visual acuity to be present in $5.52 \%$ of patients at the time of injury, which is near to the $11.5 \%$ given by Septa et al. [8]. Al-Qurainy et al. found visual acuity to be reduced in around $15 \%$ of the patients [28]. Amrith et al. reported $23 \%$ patients with reduced visual acuity, with $12.5 \%$ having permanent visual impairment [22]. In our study, 10 out of 181 patients of reduced visual acuity were having nasal bone fractures, Le Fort III fractures, frontal bone fractures, floor of the orbit fractures, and ZMC fractures. These findings suggest that reduction in visual capacity is usually associated more with higher level of complex midfacial fractures.

Total loss of vision or blindness is an uncommon sequela of maxillofacial fractures. A total of eight cases of blindness were recorded by us out of 181 patients, with the incidence being $4.42 \%$. One case was due to complete avulsion of the globe, two were due to globe rupture, two due to intraorbital hemorrhage, one due to retrobulbar neuritis, one due to traumatic optic neuropathy, one due to lens subluxation and retinal detachment, and one due to uveal, lens, and vitreous incarceration. The incidence reported in literature is between $0.3 \%$ and $3.5 \%[8,21,23,24]$. The loss of vision could be consequential to injury to the globe, optic nerve, or visual pathway.

Tearing of blood vessels at the root of the iris may lead to hyphema or blood in the anterior chamber of the eye. We found the incidence of hyphema to be $0.55 \%$ and was seen in Le Fort III fractures. The incidence relates to the findings of other studies such as those done by Septa et al. and Al-Qurainy et al., and few others, who reported it to be around $3.5 \%[8,21,23,25]$.

Traumatic telecanthus is detachment of the medial canthus of the eye. In this study, $1.66 \%$ patients presented with traumatic telecanthus, whereas the findings of the study conducted by Septa et al. showed $5 \%$ [8]. In our study, the highest incidence of traumatic telecanthus was seen in NOE fractures. 
Three (1.66\%) patients were found to have corneal tear, two (1.10\%) patients were found to have proptosis, and seven (3.87\%) patients were found to have traumatic mydriasis. The low incidence of these injuries is in accordance with the findings by Septa et al. It suggests that retinal tear, proptosis, and traumatic mydriasis are not common ocular injuries associated maxillofacial trauma [8]. Eyelid lacerations were present in 25 (13.81\%) cases, for which meticulous suturing was planned.

Al-Qurainy et al. classified the ocular injuries into mild, moderate, and severe ophthalmic injuries [21]. Among these, our study had minor ophthalmic disorders such as eyelid swelling and bruising, subconjunctival hemorrhage, chemosis, and commotio retinae (Berlin's edema). Moderate ophthalmic injuries such as enophthalmos, eyelid lacerations, traumatic pupillary changes, and lens damage were seen. Severe ophthalmic disorders included retrobulbar hemorrhage, hyphema, optic nerve injury, vitreous hemorrhage, and retinal detachment. Jamal et al. reported that $66.6 \%$ had minor ocular injuries and $10 \%$ had major ocular injuries such as ruptured globe and retinal hemorrhage [26].

In our study, ZMC fractures were associated with periorbital edema, periorbital ecchymosis, subconjunctival hemorrhage, and chemosis. Enophthalmos was seen in one case. Globe rupture and complete avulsion of the eye along with traumatic optic nerve avulsion and retrobulbar neuritis were seen in three individual cases, which led to loss of vision in these cases. All three fractures were seen in males. Exposure keratitis was also seen in one ZMC case. In the cases taken up for open reduction and internal fixation of ZMC fractures, there was no statistically significant difference between the heart rate pre-operatively and at the time of reduction of the fracture, indicating that was no significant change in the heart rate (bradycardia secondary to oculocardiac reflex during reduction of ZMC fractures) which is due to the anesthetic agents and the local infiltration of adrenaline that is given [29].

Nasal bone fractures (adding up left, right, and bilateral nasal bone fractures as shown in Table 4) accounted for the second most common midfacial fracture in our study, wherein two cases of globe rupture were seen along with other fractures leading to blindness. Canalicular injury and traumatic mydriasis were seen in two cases each, and traumatic optic neuropathy was noted in six cases. Two cases of uveal, lens, and vitreous incarceration were also seen along with nasal bone fractures.

The incidence of ocular injuries in Le Fort I fractures was very less in this study.

In association with Le Fort II fractures, three cases had traumatic optic neuropathy, one case had Berlin's edema, and one case had a corneal tear.

Le Fort III fractures were associated with a considerable number of ocular injuries ranging from subconjunctival hemorrhage, chemosis, traumatic optic neuropathy, traumatic mydriasis, and intraorbital hemorrhage.

NOE fractures presented clinically with traumatic telecanthus. Corneal tear was also diagnosed in one case of NOE fracture in our study. Frontal bone fractures also presented with a variety of ocular disorders such as diplopia, corneal tear, retrobulbar neuritis, and traumatic optic neuropathy.

Orbital fractures, especially the roof and the floor of the orbit fractures, presented with ocular injuries such as dystopia, loss of vision, intraorbital hemorrhage, and diplopia.

It is very essential to note that some of these fractures presented with some ocular injuries, which required timely management. It is necessary to not only know the common ocular injuries pertaining to a said fracture but also certain uncommon eye injuries as seen in our study.

It has been suggested that there is an increased risk of ocular injuries with maxillofacial trauma by a factor of 6.7 as compared to other major trauma patients with no facial injury [30]. The difference in the incidence of different types of ocular injuries in various studies is probably because some injuries may have gone undetected or may have been neglected in view of more significant ocular injuries also being present.

This study strives to draw attention toward concomitant ocular injuries in maxillofacial trauma patients, which may go unnoticed and later present as a serious complication incapacitating the patient. Long-term and continuous record management can make it possible to correlate ocular and orbital injures in maxillofacial trauma patients.

\section{Conclusions}

This prospective study was conducted to have an idea about concomitant orbital and ocular injuries in patients who sustained maxillofacial trauma. It is clear from this study that there is a very high probability of associated ophthalmic injuries in patients with midfacial injuries. Majority of the patients had associated eye injuries. The study stresses further on the importance of long-term and continuous data collection and record management of trauma patients, which may help health care providers with necessary information for the development of treatment protocols and device measures for the prevention of complications. The 
general population can be educated regarding the importance of obeying traffic rules and following road safety instructions with the help of such data.

\section{Additional Information \\ Disclosures}

Human subjects: Consent was obtained by all participants in this study. Research and Ethical Committee KLE VK Institute of Dental Sciences, Belagavi issued approval 1123. Animal subjects: All authors have confirmed that this study did not involve animal subjects or tissue. Conflicts of interest: In compliance with the ICMJE uniform disclosure form, all authors declare the following: Payment/services info: All authors have declared that no financial support was received from any organization for the submitted work. Financial relationships: All authors have declared that they have no financial relationships at present or within the previous three years with any organizations that might have an interest in the submitted work. Other relationships: All authors have declared that there are no other relationships or activities that could appear to have influenced the submitted work.

\section{References}

1. Salentijn EG, van den Bergh B, Forouzanfar T: A ten-year analysis of midfacial fractures . J Craniomaxillofac Surg. 2013, 41:630-636. 10.1016/j.jcms.2012.11.043

2. Motamedi MH: An assessment of maxillofacial fractures: a 5-year study of 237 patients . J Oral Maxillofac Surg. 2003, 61:61-64. 10.1053/joms.2003.50049

3. Reyes JM, Fernanda ML, Vargas GP, Rosenvasser J, Medina AJ, Funes JG: Classification and epidemiology of orbital fractures diagnosed by computed tomography. Rev Argent Radiol. 2013, 77:139-146.

4. Patil SG, Kotwal IA, Joshi U, Allurkar S, Thakur N, Aftab A: Ophthalmological evaluation by a maxillofacial surgeon and an ophthalmologist in assessing the damage to the orbital contents in midfacial fractures: a prospective study. J Maxillofac Oral Surg. 2016, 15:328-335. 10.1007/s12663-015-0844-8

5. Mittal G, Singh N, Suvarana S, Mittal SR: A prospective study on ophthalmic injuries related to maxillofacial trauma in Indian population. Natl J Maxillofac Surg. 2012, 3:152-158. 10.4103/0975-5950.111370

6. Mehravaran R, Akbarian G, Nezhad CM, Gheisari R, Ziaei M, Zadeh FG: Evaluation of the relationship between the pattern of midfacial fractures and amaurosis in patients with facial trauma. J Oral Maxillofac Surg. 2013, 71:1059-1062. 10.1016/j.joms.2013.01.007

7. Ansari MH: Blindness after facial fractures: a 19-year retrospective study . J Oral Maxillofac Surg. 2005, 63:229-237. 10.1016/j.joms.2004.05.221

8. Septa D, Newaskar VP, Agrawal D, Tibra S: Etiology, incidence and patterns of mid-face fractures and associated ocular injuries. J Maxillofac Oral Surg. 2014, 13:115-119. 10.1007/s12663-012-0452-9

9. Vetter JD, Topazian RG, Goldberg MH, Smith DG: Facial fractures occurring in a medium-sized metropolitan area: recent trends. Int J Oral Maxillofac Surg. 1991, 20:214-216. 10.1016/s0901-5027(05)80177-8

10. Zachariades N, Papavassiliou D: The pattern and aetiology of maxillofacial injuries in Greece: a retrospective study of 25 years and a comparison with other countries. J Craniomaxillofac Surg. 1990, 18:251-254. 10.1016/s1010-5182(05)80425-1

11. Fridrich KL, Pena-Velasco G, Olson RA: Changing trends with mandibular fractures: a review of 1,067 cases . J Oral Maxillofac Surg. 1992, 50:586-589. 10.1016/0278-2391(92)90438-6

12. Olasoji HO, Tahir A, Arotiba GT: Changing picture of facial fractures in northern Nigeria . Br J Oral Maxillofac Surg. 2002, 40:140-143. 10.1054/bjom.2001.0716

13. Lalani Z, Bonanthaya KM: Cervical spine injury in maxillofacial trauma. Br J Oral Maxillofac Surg. 1997, 35:243-245. 10.1016/s0266-4356(97)90041-3

14. Rahman RA, Ramli R, Rahman NA, Hussaini HM, Idrus SM, Hamid AL: Maxillofacial trauma of pediatric patients in Malaysia: a retrospective study from 1999 to 2001 in three hospitals. Int J Pediatr Otorhinolaryngol. 2007, 71:929-936. 10.1016/j.ijporl.2007.03.003

15. Mwaniki DL, Guthua SW: Occurrence and characteristics of mandibular fractures in Nairobi, Kenya . Br J Oral Maxillofac Surg. 1990, 28:200-202. 10.1016/0266-4356(90)90089-4

16. Bamjee Y, Lownie JF, Cleaton-Jones PE, Lownie MA: Maxillofacial injuries in a group of South Africans under 18 years of age. Br J Oral Maxillofac Surg. 1996, 34:298-302. 10.1016/s0266-4356(96)90006-6

17. Ashar A, Kovacs A, Khan S, Hakim J: Blindness associated with midfacial fractures . J Oral Maxillofac Surg. 1998, 56:1146-1151. 10.1016/s0278-2391(98)90757-1

18. Al Ahmed HE, Jaber MA, Abu Fanas SH, Karas M: The pattern of maxillofacial fractures in Sharjah, United Arab Emirates: a review of 230 cases. Oral Surg Oral Med Oral Pathol Oral Radiol Endod. 2004, 98:166-170. 10.1016/j.tripleo.2004.01.020

19. Erol B, Tanrikulu R, Görgün B: Maxillofacial fractures. Analysis of demographic distribution and treatment in 2901 patients (25-year experience). J Craniomaxillofac Surg. 2004, 32:308-313. 10.1016/j.jcms.2004.04.006

20. Bataineh AB: Etiology and incidence of maxillofacial fractures in the north of Jordan . Oral Surg Oral Med Oral Pathol Oral Radiol Endod. 1998, 86:31-35. 10.1016/s1079-2104(98)90146-9

21. Al-Qurainy IA, Stassen LF, Dutton GN, Moos KF, El-Attar A: The characteristics of midfacial fractures and the association with ocular injury: a prospective study. Br J Oral Maxillofac Surg. 1991, 29:291-301. 10.1016/0266-4356(91)90114-k

22. Amrith S, Saw SM, Lim TC, Lee TK: Ophthalmic involvement in cranio-facial trauma. J Craniomaxillofac Surg. 2000, 28:140-147. 10.1054/jcms.2000.0138

23. Marín MI, Tejero TR, Dominguez FM, Gutiérrez ME: Ocular injuries in midfacial fractures . Orbit. 1998, 17:41-46. 10.1076/orbi.17.1.41.7950

24. Lim LH, Lam LK, Moore MH, Trott JA, David DJ: Associated injuries in facial fractures: review of 839 


\section{Cureus}

patients. Br J Plast Surg. 1993, 46:635-638. 10.1016/0007-1226(93)90191-d

25. Barry C, Coyle M, Idrees Z, Dwyer MH, Kearns G: Ocular findings in patients with orbitozygomatic complex fractures: a retrospective study. J Oral Maxillofac Surg. 2008, 66:888-892. 10.1016/j.joms.2008.01.005

26. Jamal BT, Pfahler SM, Lane KA, et al.: Ophthalmic injuries in patients with zygomaticomaxillary complex fractures requiring surgical repair. J Oral Maxillofac Surg. 2009, 67:986-989. 10.1016/j.joms.2008.12.035

27. Cheema SA, Amin F: Incidence and causes of maxillofacial skeletal injuries at the Mayo Hospital in Lahore, Pakistan. Br J Oral Maxillofac Surg. 2006, 44:232-234. 10.1016/j.bjoms.2005.05.017

28. Al-Qurainy IA, Stassen LF, Dutton GN, Moos KF, El-Attar A: Diplopia following midfacial fractures. Br J Oral Maxillofac Surg. 1991, 29:302-307. 10.1016/0266-4356(91)90115-1

29. Sharma R, Chhabra N, Sharma P, Chhabra S: Evaluation of intra ocular pressure in zygomatico maxillary complex fractures. J Maxillofac Oral Surg. 2015, 14:226-233. 10.1007/s12663-013-0614-4

30. Guly CM, Guly HR, Bouamra O, Gray RH, Lecky FE: Ocular injuries in patients with major trauma. Emerg Med J. 2006, 23:915-917. 10.1136/emj.2006.038562 